\title{
The NEID spectrometer: fibre injection system design
}

Schwab, Christian, Monson, Andrew, Kanodia, Shubham, Lubar, Emily, Lin, Andrea, et al.

Christian Schwab, Andrew J. Monson, Shubham Kanodia, Emily Lubar, Andrea J. Lin, Colin Nitroy, Samuel Halverson, Qian Gong, Ryan C. Terrien, Joe P. Ninan, Chad Bender, Cullen H. Blake, Frederick R. Hearty, Suvrath Mahadevan, Michael W. McElwain, Paul M. Robertson, Arpita Roy, Gudmundur Stefansson, "The NEID spectrometer: fibre injection system design," Proc. SPIE 11447, Ground-based and Airborne Instrumentation for Astronomy VIII, 114474L (13 December 2020); doi: 10.1117/12.2563248 


\title{
The NEID spectrometer: fibre injection system design
}

\author{
Christian Schwab*a, Andrew J. Monson ${ }^{\mathrm{b}}$, Shubham Kanodia ${ }^{\mathrm{b}}$, Emily Lubar ${ }^{\mathrm{b}}$, \\ Andrea S. J. Lin ${ }^{\mathrm{b}}$, Colin Nitroy ${ }^{\mathrm{b}}$, Samuel Halverson ${ }^{\mathrm{c}}$, Qian Gong ${ }^{\mathrm{d}}$, Ryan C. Terrien ${ }^{\mathrm{e}}$, \\ Joe P. Ninan ${ }^{\mathrm{b}}$, Chad Bender ${ }^{\mathrm{f}}$, Cullen Blake ${ }^{\mathrm{g}}$, Frederick R. Hearty ${ }^{\mathrm{b}}$, Suvrath Mahadevan ${ }^{\mathrm{b}}$, \\ Michael W. McElwain ${ }^{\mathrm{d}}$, Paul M. Robertson ${ }^{\mathrm{h}}$, Arpita Roy ${ }^{\mathrm{i}}$, and Gudmundur Stefansson ${ }^{\mathrm{j}}$ \\ aDepartment of Physics \& Astronomy, Macquarie University, NSW 2109, Australia; \\ ${ }^{b}$ Department of Astronomy \& Astrophysics, The Pennsylvania State University, University Park, \\ USA, 16802; ' 'Jet Propulsion Laboratory, Pasadena, CA 91109, USA; d NASA Goddard Space Flight \\ Center, Greenbelt, MD, 20771, USA; ${ }^{\mathrm{e} C a r l e t o n}$ College, Northfield, MN, 55057, USA; ${ }^{\mathrm{f}}$ Steward \\ Observatory, University of Arizona, Tucson, AZ 85719; ' $D$ Department of Physics \& Astronomy, \\ University of Pennsylvania, Philadelphia, PA, USA, 19104; ${ }^{\text {h}}$ Department of Physics \& Astronomy, \\ The University of California, Irvine, CA USA; iSpace Telescope Science Institute, Baltimore, MD, \\ 21218, USA, ${ }^{\mathrm{j} P r i n c e t o n}$ University, Princeton, NJ 08540, USA
}

\begin{abstract}
NEID is a high resolution echelle spectrograph designed to enable extremely precise Doppler radial velocity observations of stars in the $380-930 \mathrm{~nm}$ wavelength range ${ }^{1}$. It has recently been installed at the $3.5 \mathrm{~m}$ WIYN telescope at Kitt Peak National Observatory, and is currently being commissioned. The design is based on a white pupil layout with a monolithic parabolic primary mirror and a $195 \mathrm{~mm}$ pupil size on the R4 Echelle grating. Here we describe the optical and mechanical design, assembly, and alignment of the fiber injection system which converts the native focal ratio of the sky, calibration, and science fibers to the focal ratio required to form the $195 \mathrm{~mm}$ collimated beam.
\end{abstract}

Keywords: Echelle spectroscopy, Exoplanets. Doppler technique, Radial Velocities, Fiber injection, Spectrograph, High-resolution spectroscopy

\section{INTRODUCTION}

Large echelle spectrographs for precision radial velocity (PRV) observations are highly optimised optical systems with very particular requirements. The most common implementation uses a so-called white pupil relay (WPR) illuminating a large echelle grating as main dispersive element, and either a prism, or a low-dispersion grating or grism as a cross disperser placed in the pupils of the relay with a collimated beam $^{2}$. The optical design typically focuses on the spectrograph camera(s) which image the two-dimensional spectrum at the exit of the mirror-based relay system onto a detector. These cameras are often large, complex and, consequently, costly. The fiber injection optics (sometimes labelled FN-system) form a much smaller subassembly; however, they also require careful consideration as they impact the image quality, throughput, and potentially stability, of the whole instrument.

The role of the fiber injection system is primarily to adapt the numerical aperture of the fibers which feed the spectrograph to the appropriate focal ratio of the collimator (typically an off-axis parabola). This generally requires the beam from the fibers to be slowed down. For simultaneous calibration, sky subtraction or image slicing, multiple fibers are fed into the spectrograph which all need to be imaged with appropriate image quality. In the case of NEID, three fibers (in the high resolution mode) with exit f-ratios of $\sim 3.6$ need to be projected onto the collimator with an f-ratio of $7.95^{3}$.

The stability of the fiber injection system, in an absolute sense, and in particular that of the fibers with respect to each other, is of outmost importance for the RV precision the instrument can achieve ${ }^{4}$. Any differential movement between the fibers, or respectively their images, cannot be removed with a calibration spectrum taken through a calibration fiber. In NEID, all fibers are glued in a custom-made fused silica puck that places their apertures in precisely the desired positions relative to each other, as well as stably maintaining them in that same location.

*Send correspondence to C.S.: christian.schwab@mq.edu.au; phone +61 298506288 


\section{REQUIREMENTS}

The technical requirements that drive the optomechanical design of the fiber-reimaging optics were derived from the top level requirements that underpin the performance needed to address the primary science of the instrument, and the radial velocity error budget we developed for $\mathrm{NEID}^{4}$. The driving requirements were:

- High throughput, $>90 \%$ across the full wavelength range (380-930nm)

- $\mathrm{f} / 7.95$ exit focal ratio

- $\quad 0.2 \mathrm{~mm}$ object field (to accommodate the three high-resolution (HR) and two high efficiency (HE) fibers

- Small wavefront error (< lambda/25 RMS at all wavelengths and field points)

- Achievable alignment tolerances

- Accessible pupil for straylight suppression

- Near telecentric to avoid beam clipping

- Compact to be compatible with the mechanical constraints

The injection optics need to convert the fiber's output from $\mathrm{f} / 3.6$, which takes into account some FRD expected in our fiber link, to the required focal ratio of the spectrograph's main collimator, to achieve a collimated beam diameter of $195 \mathrm{~mm}$. With a given focal length for the collimator mirror of $1550 \mathrm{~mm}$, this yields an output $\mathrm{f} / \mathrm{ratio}$ of $\mathrm{f} / 7.95$ of the fiber injection system towards the parabolic mirror. The total field on the input side is $+/-0.2 \mathrm{~mm}$ high in cross-dispersion direction (centers of fibers at $[0,0]$ and $[0,+/-0.17] \mathrm{mm}$ ). The field in dispersion direction is $0.25 \mathrm{~mm}$ wide. The system needs to be highly transmissive over the whole spectral range into the UV, so the use of i-Line or high transmission glasses and to restrict the number of optical surfaces is important.

Excellent image quality (respectively, a flat wavefront of the collimated beam generated by the injection system plus collimator) is obviously important to avoid degrading the spectrograph's resolution. It is important to note that even small aberrations in the beam are exacerbated by the grating and the prism, which, for example, generate significant linear astigmatism from slightly converging or diverging beams, and generally produce higher aberrations when illuminated with non-flat wavefronts. In addition to the loss of resolution, another, more subtle effect is important for PRV work. Spots enlarged by aberrations are more susceptible to changes in pupil illumination than smaller spots. Large spots effectively map the light from different parts of the pupil onto different parts of the point spread function (PSF). If the relative illumination of different parts of the pupil changes, so does the PSF, and its barycentre shifts, which leads to spurious RV shifts. A perfect, diffraction limited PSF shifts much less due to pupil illumination instability than a PSF with large wings due to low-order aberrations. This is the reason for the significant effort by many groups on improving fiber scrambling in the near- and far-field (pupil), to stabilize the illumination of the spectrograph optics ${ }^{5,6,7}$. Small PSFs that approach the diffraction limit can significantly help in desensitizing the spectrograph from remaining pupil illumination variations, which aids in easing the challenging requirements on the scrambling gain of the fiber feed. For NEID's injection optics, we aimed for a design wavefront error of less than lambda/25 RMS at any field point and wavelength.

The input of the injection system is telecentric by definition, as it is fed by parallel fibers which generate parallel cones of light. We require the beam footprints on the grating from the different fibers (field points) to not be displaced with respect to each other, to avoid light loss and mode clipping on the aperture stop of the grating or elsewhere. This requires the injection system to be (near) telecentric on the output. As a working definition, we consider a beam displacement of $<1 \%$ of the total beam size, or $2 \mathrm{~mm}$, on a dummy surface perpendicular to the beam and at the location of the grating's vertex acceptable.

We ideally want an internal pupil plane in the injection system that is accessible, to mount a pupil stop to limit the imperfectly defined beam from the fiber to the nominal diameter and avoid light outside this footprint, where it could illuminate optics mounts and produce straylight. In practical terms, this aperture, which is necessarily small, would require tight mechanical tolerances and a well-defined, achromatic pupil image to be used as the actual pupil stop, that is, the limiting aperture setting the beam diameter. As is later shown, the beam diameter in the injection optics is about $2 \mathrm{~mm}$, or $1 \%$ of the beam diameter on the grating. For the desired straylight suppression, a slightly oversized baffle $(+0.1 \mathrm{~mm}$, leading to a $\sim 10 \mathrm{~mm}$ oversized projected aperture on the grating) is sufficient, and reduces the requirement on the injection optics and mechanics. NEID also has a blackened, elliptical baffle a few millimetres above the echelle grating's surface, which more precisely defines the transmitted beam size. 


\section{DESIGN APPROACH}

It is useful to consider the specific geometry of a typical injection setup in a spectrograph based on a white pupil relay (WPR) using an off-axis parabola to collimate the light. In NEID, we use a single monolithic parabolic mirror for the WPR. We use an off-axis portion of this mirror as OAP (off-axis parabola) collimator. The off-axis angle is 5 degrees in cross dispersion direction. The light is injected at that angle by rotating the injection system as a rigid body; this leads to a tilt of the fiber surface plane with respect to the optical axis of the parabola, which is the surface normal of the parent parabola's vertex. In consequence, the off-axis fibers are located slightly in front of and behind the focus, respectively. We have investigated whether this should be corrected by wedging the lens elements in the injection system; in practice, this was found to be not necessary due to the small input field size. The other aberration inherent in the setup is off axis coma of the fast parabola. To account for this, the injection systems were all optimized with the collimator mirror in place, and a paraxial lens in place of the Echelle grating (see Fig. 1). We thereby optimize the beam quality on the Echelle grating, balancing the defocus and coma of the main mirror against the aberrations of the injection optics.

The simplest way to convert the fibers' f-ratio to the desired value would be a positive lens mounted within one focal length to the fibers, generating a virtual focus behind the fibres with a slower beam. However, this leads to an unacceptable location of the pupil, and significant beam displacement on the grating. While it requires significantly more optical power, this is the main reason to use a system that generates a real focus. As a minor point, this also helps with the mechanical design, as it places the injection optics behind the fold mirror (sometimes called spectrum mirror) of the WPR.

We used different starting points in Zemax for the optics and then did a trade-off between their performance. We used initial systems with and without a plano-convex singlet lens in contact with the fiber. This was followed by a pair of airspaced or cemented doublets, a pair of cemented triplets, and a single triplet. We constrained the lens parameters (minimum radii, maximum diameters and thickness) to ensure the optics can be manufactured. Glass optimization was carried out through Hammer and Global Optimization algorithms, using custom glass catalogs selected for high-transmission glass types (predominantly i-Line glasses and a few additional types from the Ohara, Schott and Nikon catalogs). We chose to not enforce correction of lateral color, as this places additional constraints on the lens system, and the wavelengths are separated out by the spectrograph's dispersive elements in any case. However, this leads to a change in the way the order spacing, as well as effective magnification of the injection system relay, varies with wavelength, leading to a wavelengthdependent change in resolution and sampling. We took care to ensure these effects stayed within acceptable limits.

The use of the plano-convex singlet cemented to the fiber apertures is a convenient way to slow down the beam from the fibers significantly (on the order of the refractive index of the lens) with very little added aberrations. This can be easily understood by considering the case of one single fiber and a plano-convex lens, with the flat side in contact with the fiber, and the convex side concentric to the fiber. In this case, the rays emerging from the fiber travel uninterrupted into the lens; that is, without encountering a glass-air surface, and without being refracted away from the optical axis of the fiber. The beam stays at the slower focal ratio it has inside the fiber ( a factor $n$ ). At the surface of the lens, all rays are perpendicular to the surface, as it is concentric with the fiber, and again the rays are not refracted, but keep traveling straight into the air. This arrangement has basically no cost in throughput, as the number of glass-air surfaces stays the same compared to a bare fiber. Indeed, in this way, one can avoid a complicated AR coating on the fiber cable, which instead is applied much more easily to the lens.

In the merit function driving the optimisation, we evaluated the RMS spot size in the focal plane of the paraxial camera we put in place of the echelle. We optimized each initial lens configuration for about one week on a desktop PC, and then compared the results for image quality (wavefront error), throughput, pupil displacement for the outer fibers on the grating, and location and chromatic variation of the internal pupil.

As a final step, we inserted the injection optics models into the full optical model of the spectrograph including the real camera and cross-disperser prism, to verify the performance of the full system. Specifically, we evaluated the achieved resolution across the detector in comparison to an ideal injection system by convolving the unaberrated fiber image with the local PSF, and measuring the resulting FWHM of a monochromatic line. 


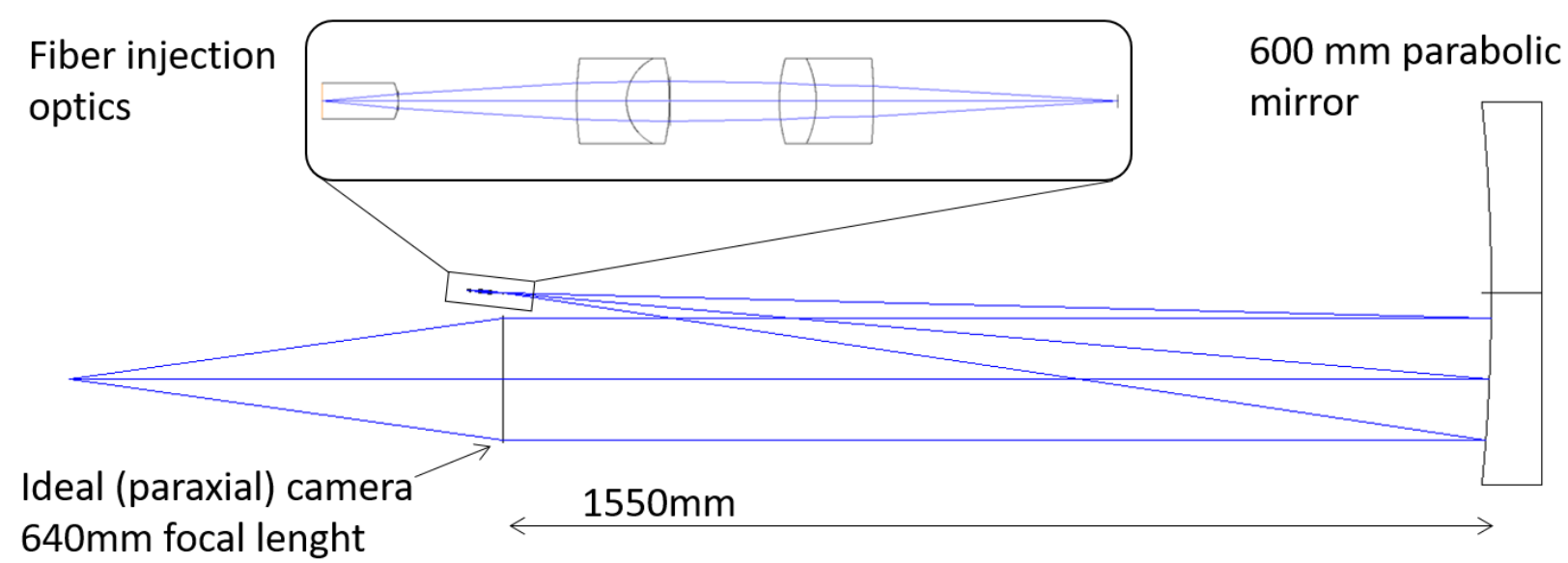

Figure 1. Layout of the model system used to optimize the injection optics. The injection optics are located in the focus of a parabolic mirror with $1550 \mathrm{~mm}$ focal length, as the primary mirror of NEID. They are tilted by 5 degrees w.r.t the optical axis of the mirror. In the pupil plane of the mirror, where the echelle grating is placed in the full spectrograph model, we put a paraxial camera with the same focal length as the NEID camera, to generate spots and evaluate the image quality of the complete system of injection optics and parabolic mirror.

\section{DESIGN VARIANTS}

For reference, we made a design which allowed wedged elements, to account for the broken symmetry of the off-axis parabola setup. In this design, we used a singlet cemented to the fiber, followed by a cemented triplet. We found that this did not offer any practical improvement over a similar, but rotationally symmetric design, at much increased complexity. We therefore discarded those design options.

As a second reference point, we looked at a fully telecentric design that allowed crystal materials $(\mathrm{CaF} 2)$ and used to airgap doublets (dubbed Q1). This design uses two confocal, air-spaced doublets, with CaF2 as the positive element in both, and with a collimated space between them, which also contains a pupil. This design delivers diffraction limited image quality, measured as described above. Lateral colour is small, but not fully corrected. We consider the airgaps in the doublets, which are small and between steeply curved surfaces, critical to align, resulting in tight internal centration tolerances for the doublets. The lenses are $4 \mathrm{~mm}$ in diameter.

Because of the tight tolerances and the use of $\mathrm{CaF} 2$ we consecutively moved on to three alternative designs:

C2: using two cemented triplets instead of the two air-spaced doublets;

C3: replacing the triplets with cemented doublets and introducing a singlet glued to the fibers

C4: using only one triplet, and a singlet glued to the fibers.

All these designs, once optimized, were able to provide diffraction limited performance across the full spectral grasp of NEID (considered separately at each wavelength). They do differ in spot size, performance over the field, throughput, alignment sensitivity, pupil position and fidelity, and overall complexity. Below, we briefly present the performance for each design by showing the layout of the lens system, the resulting spots, and discuss its specifications. A summary of the results is given in Table 1 after presenting the design variants. Configurations other than those we investigated here are of course possible, from different lens and lens group configurations to reflective designs. We also limited the physical size of the designs we considered to match our practical constraints on space in the very tight volume between the echelle grating mount and the fold mirror in the focal plane of the parabolic mirror (next to the injection optics). 


\subsection{Design Q1}

This design uses two air-spaced doublets, has the best correction of lateral color (near perfect), and provides the most precisely defined pupil of all the ones discussed here. Its transmission is lowest, due to the 8 glass-air surfaces, and alignment is likely critical within each group, due to the tight airgap. Below the layout is shown in Figure 2, and a matrix spot diagram depicting the geometric spots generated in the focal plane of the paraxial camera for all wavelengths and four different field heights is shown in Figure 3.

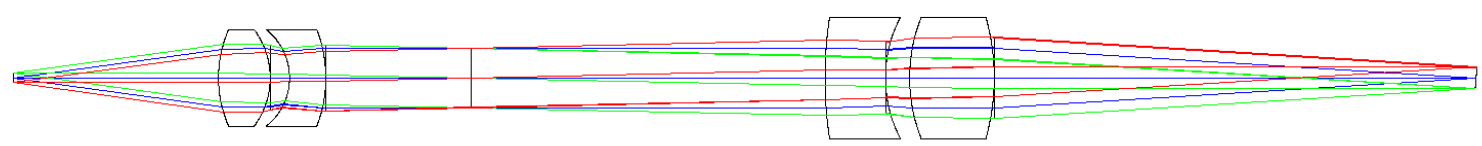

Figure 2. Layout of the injection system for design Q1; fibers on the left side. The lens diameter is 4mm, the glass types are CaF2, PBL25Y, and PBL6Y (Ohara).

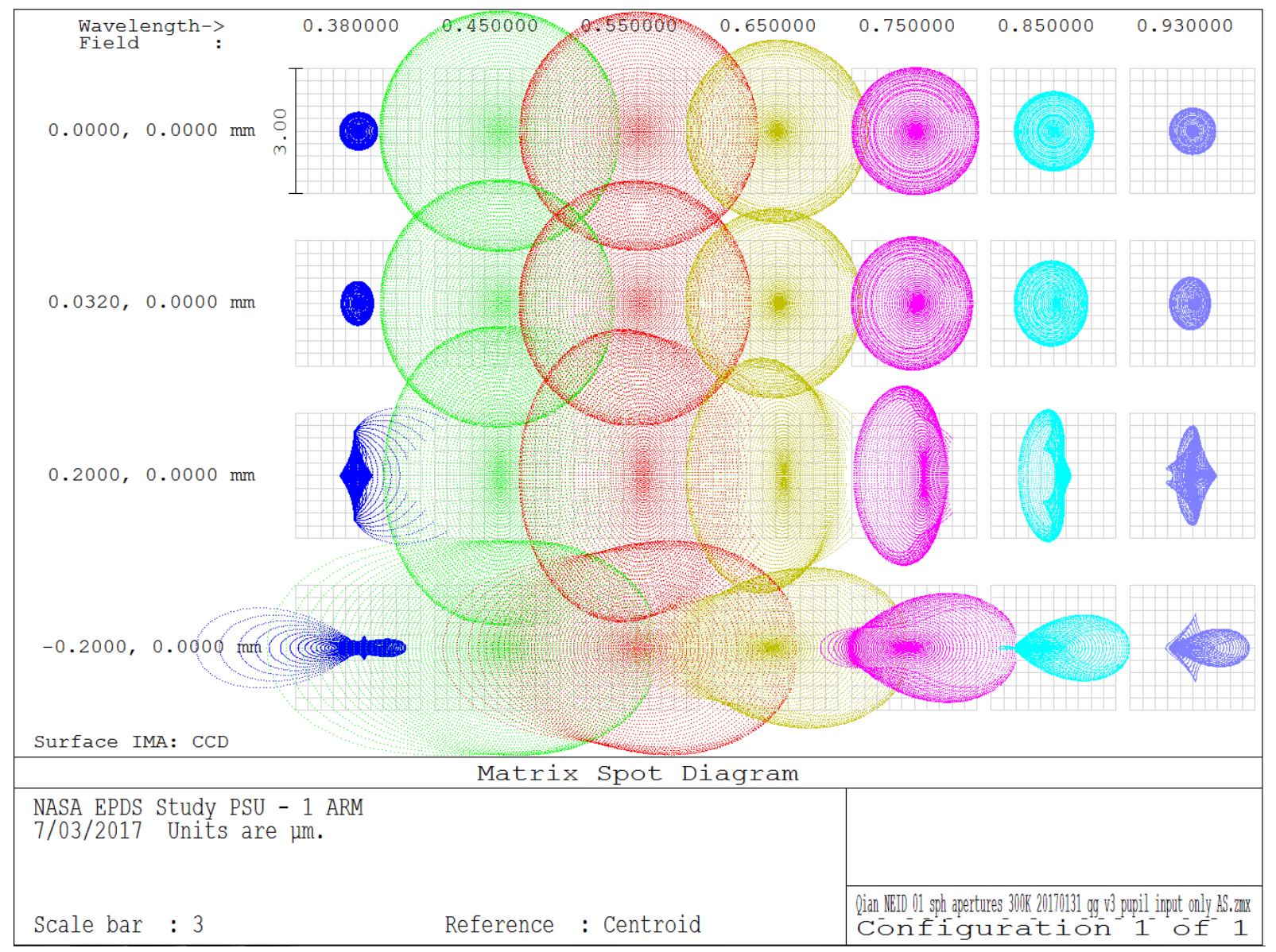

Figure 3. Matrix spot diagram showing the spot quality measured in the focal plane of the paraxial camera for design Q1 as function of 8 different wavelengths from $380 \mathrm{~nm}$ to $930 \mathrm{~nm}$, and for the central field, the edge of the central fiber, and the outer edge of the sky and calibration fiber. The box sizes are 3 microns (compared to our pixel size of 10 microns, and an Airy disc diameter of $\sim 4.5$ microns). 


\subsection{Design C2}

To improve upon throughput by removing any air-glass surfaces possible, and to mitigate the alignment tolerances of the small airgaps, we calculated a design with cemented triplets in place of the air-spaced doublets. For manufacturing reasons, we excluded crystal materials from the glass catalog. Layout and spots are shown below.

This design improves color correction and spot size by a significant margin. The cemented triplets use fluorophosphate glasses (S-FPL53 and S-FPL51Y) as center elements, which makes these elements from glasses that are more challenging to work with easier to polish and avoids coating them. The matching elements on the outside of the triplets are durable crown and flint glasses. Internal radii are comparable to the radii in the $\mathrm{CaF} 2$ doublets from Q1, but as they are cemented, are easier to produce in a quality that enables very low wavefront error. Importantly, losses are a factor of two lower, as the total number of glass-air interfaces drops from 8 to 4 . The outside radii of the triplets are very moderate, leading to improved alignment tolerances. This design is also nearly fully corrected for lateral color.

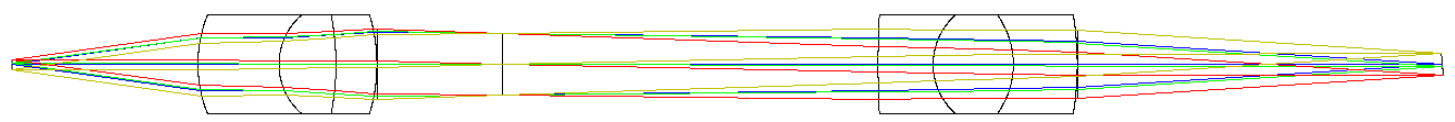

Figure 4. 3D layout of the cemented triplet design. Lens diameter is 4mm. The glasses used are (PBM8Y, S-FPL53, PBL26Y) and (N-BK10, S-FPL51Y, N-BK10).

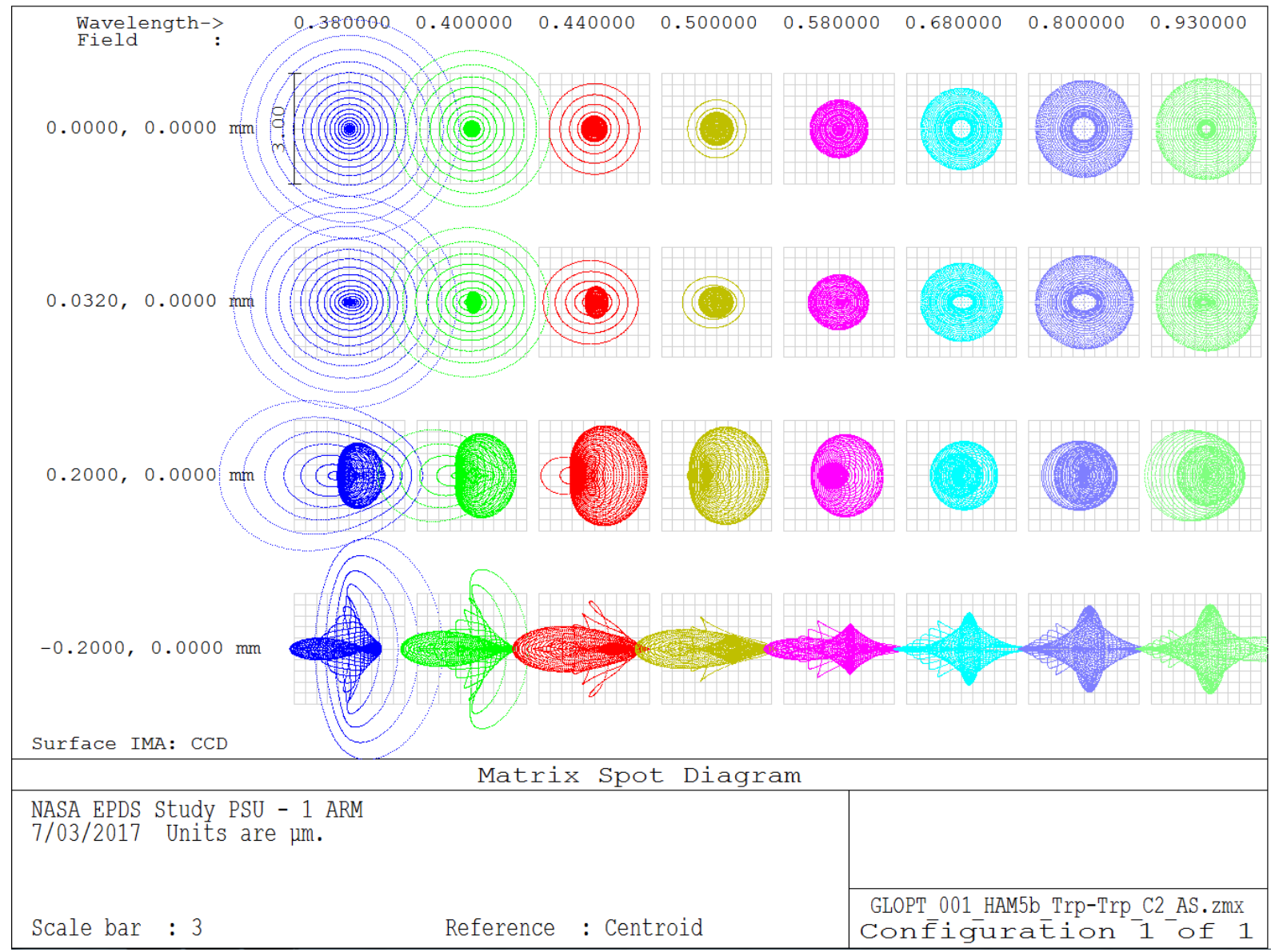

Figure 4. Matrix spot diagram of the cemented triplet design. Scale and wavelength range identical to the equivalent plot above. 


\subsection{Design C3}

As the next version, we replaced the triplets with doublets, and inserted a plano-convex singlet lens which is glued onto the fibers. We required the glass for this lens to be fused silica, to match the coefficient of thermal expansion of the custom fused silica puck that holds the fibers.

The singlet in contact with the fiber improves the image quality and corrected field of view of this design significantly. If this lens were made concentric with the fiber, it would keep our beam at a moderately slow $f / 5.2$, the focal ratio the beam has in glass (instead of f/3.6 in air). In practice, when optimizing the injection system with the convex lens surface's radius and thickness as given, the lens provides more power than in the concentric case, and assumes a shorter radius, resulting in an even slower beam entering the first doublet. We have investigated solutions with different glasses for the singlet, and find, unsurprisingly, that low dispersion glasses seem to work well. However, the gain in performance compared to fused silica is minor, and we chose to select fused silica to match the fibers and fiber puck material.

With the singlet added, the two cemented doublets provide excellent image quality surpassing all the previously described systems. However, the pupil now lies between the singlet and the first doublet, and lateral chromatic aberration becomes noticeable.

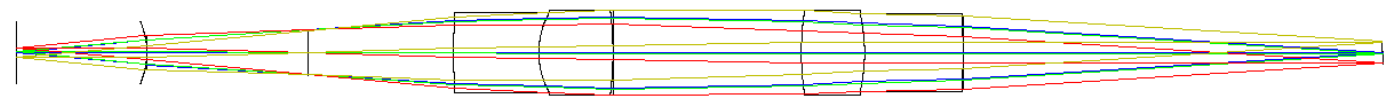

Figure 5. Glasses in the doublets are (N-BK10, S-FPL51Y) and (S-FPL53, NIGS5742). Shown is only the lens diameter at the clear aperture, which is $3.4 \mathrm{~mm}$.

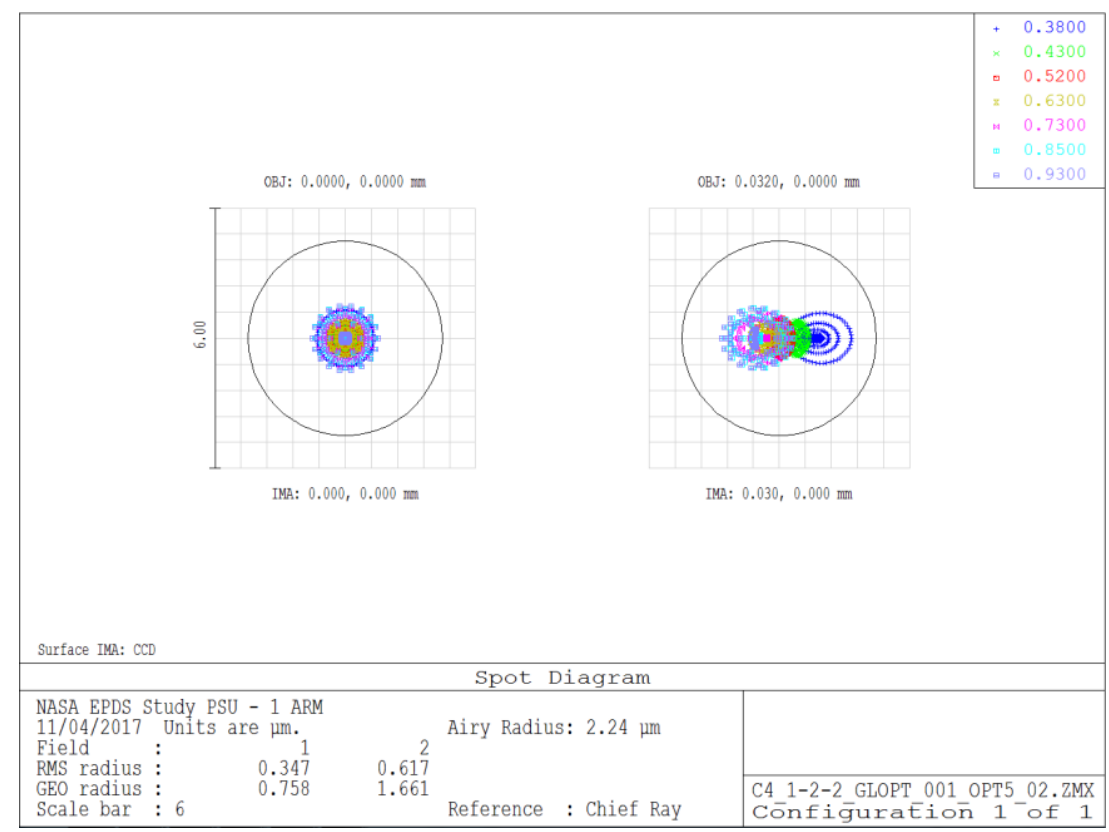

Figure 6. The polychromatic spot diagrams for the center and edge of the science fiber show the small amount of lateral color in this design and illustrate the diffraction limited performance. The circle shows the Airy disc. 


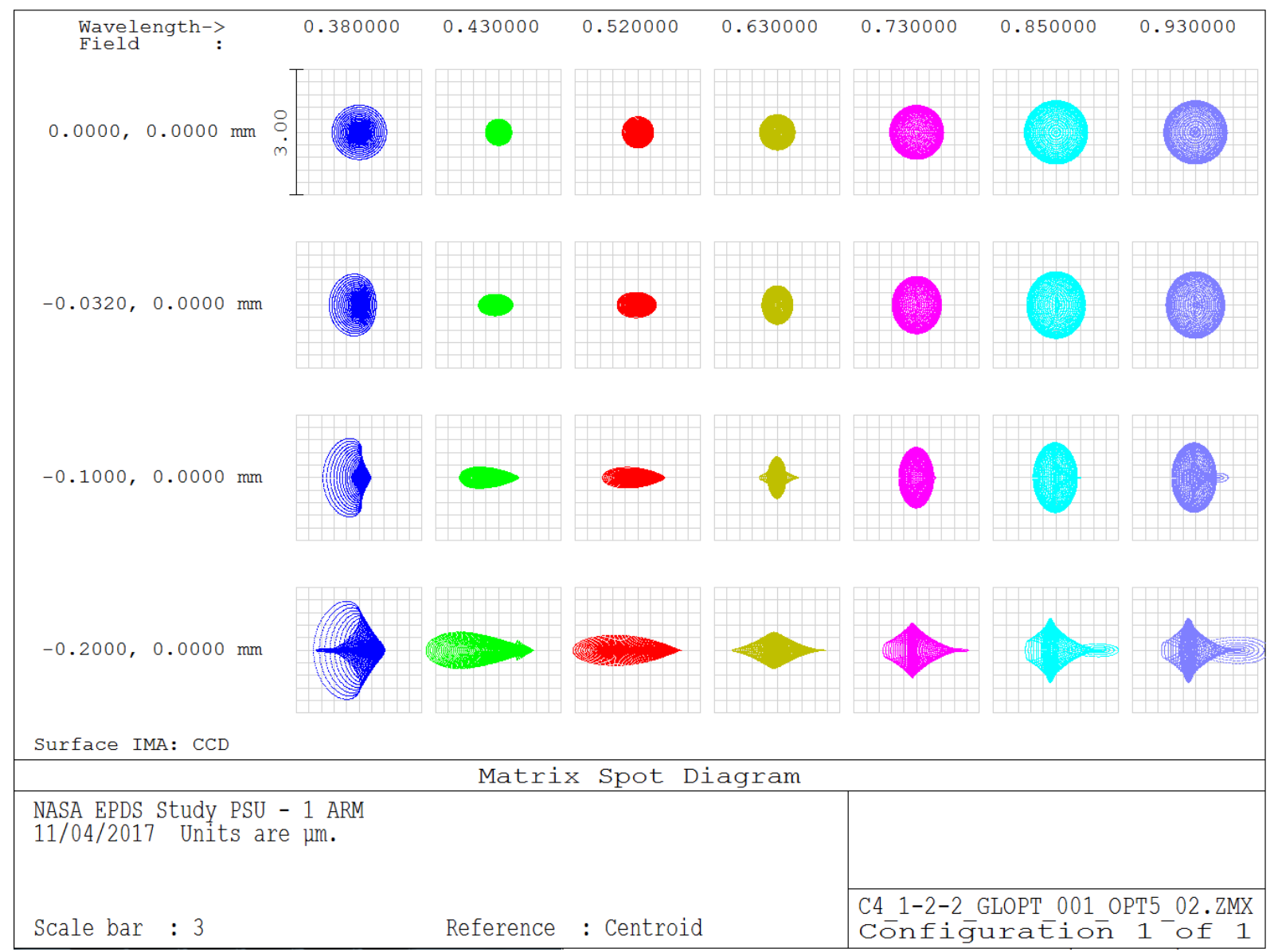

Figure 7. Matrix spot diagram. This design clearly shows excellent image quality at all wavelengths and across the full object field.

\subsection{Design $\mathrm{C4}$}

The last design variant we investigated was a singlet on the fiber and a single cemented triplet to refocus the beam at the desired f-ratio of $\mathrm{f} / 7.95$. For design $\mathrm{C} 4$, we constrained the lens radii to stay above a certain value to ensure manufacturing is possible, and we investigated versions with the singlet material fixed to fused silica, as well as versions with the singlet's material to be taken from a custom catalogue of high transmission glasses at $380 \mathrm{~nm}$. The image quality naturally is slightly worse than design $\mathrm{C} 3$. At the same time, $\mathrm{C} 4$ provides the highest possible throughput with only two extra glass-air surfaces beyond the fiber exit. The pupil lies between the singlet and the triplet. The lens radii, while steeper, are moderate on the glass-air surfaces ( $\mathrm{f} / 2$ if they were mirrors), yielding acceptable alignment tolerances for the triplet as a group. This design produces a small amount of lateral color, which leads to a wavelength dependent magnification of the fiber, as well as varying beam diameter and added cross dispersion.

These effects are at the few percent level.

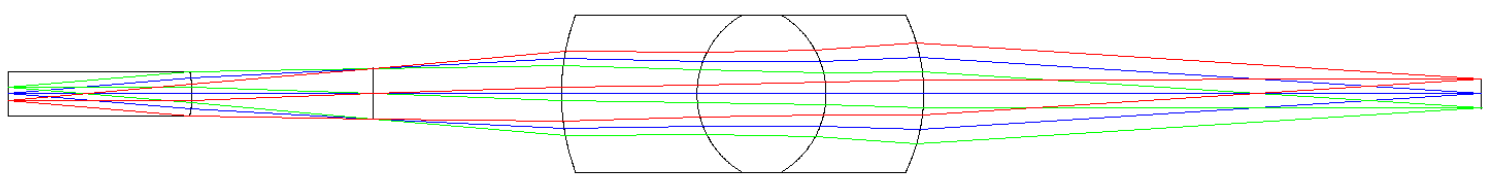

Figure 8. Layout of design C4, singlet plus cemented triplet. The pupil is located before the triplet lens. The lens diameter is 4mm, the glasses used are PBM8Y for the singlet, and (BSL7Y, S-FPL55, PBL25Y) for the triplet. 


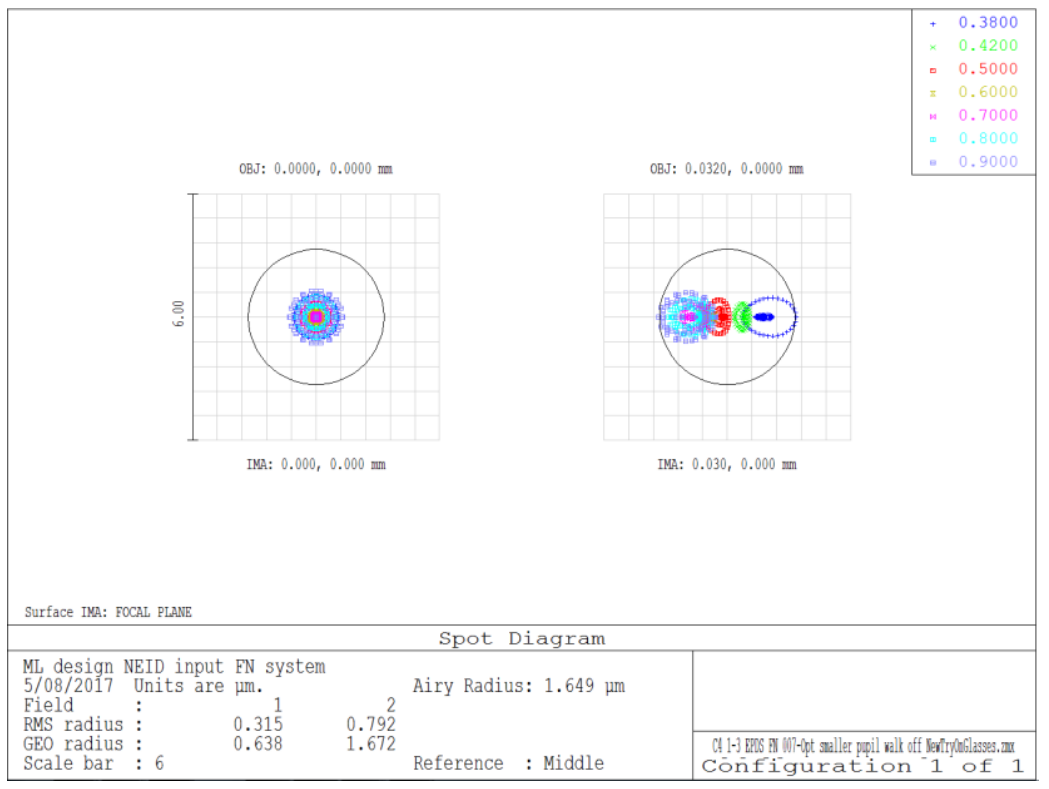

Figure 9. Spots at field center and the edge of the science fiber. This design exhibits a moderate amount of lateral color, which leads to a variable fiber magnification ( $<3 \%$ variation across the full wavelength range).

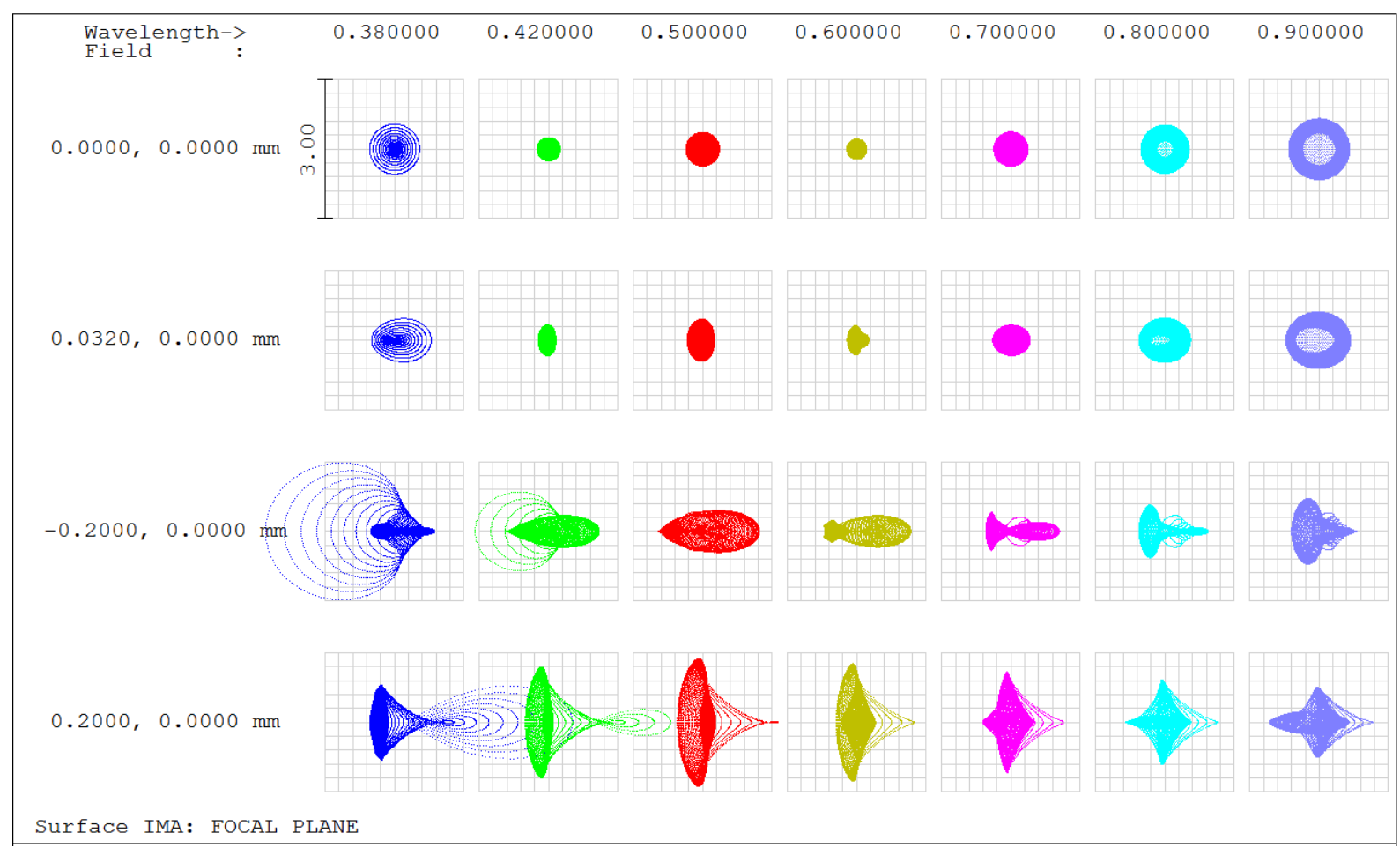

Figure 10. Matrix spot diagram. Image quality is excellent in the center, and drops off somewhat towards the outer fibers, and at the bluest end of the wavelength range.

\subsection{Design comparison table}

Below we list the key performance specifications of the designs presented above. Based on this assessment, and after consultation with vendors about manufacturing options, we selected design C3, using a singlet on the fibers followed by two cemented doublets, for the injection system of NEID. 
Table 1. Performance comparison between different designs.

\begin{tabular}{|l|l|l|l|l|}
\hline Design & Q1 & C2 & C3 & C4 \\
\hline On axis spot radius (micron RMS) & $1.36 \mu \mathrm{m}$ & $0.82 \mu \mathrm{m}$ & $0.35 \mu \mathrm{m}$ & $0.32 \mu \mathrm{m}$ \\
\hline $\begin{array}{l}\text { Off axis RMS spot radius at 380nm } \\
\text { (0.2mm field) }\end{array}$ & $\begin{array}{l}2.5 \mu \mathrm{m} \\
(@ 450 \mathrm{~nm})\end{array}$ & $1.30 \mu \mathrm{m}$ & $0.59 \mu \mathrm{m}$ & $1.84 \mu \mathrm{m}$ \\
\hline $\begin{array}{l}\text { Lateral color (image height variation of } \\
\begin{array}{l}\text { 0.2mm field point - variation in order } \\
\text { spacing) }\end{array}\end{array}$ & $0 \%$ & $1.8 \%$ & $4.4 \%$ & $6.4 \%$ \\
\hline $\begin{array}{l}\text { Lateral color (image height variation of } \\
\begin{array}{l}\text { 0.032mm field point - variation in } \\
\text { resolution) }\end{array}\end{array}$ & $0 \%$ & $1.7 \%$ & $4.4 \%$ & $6.3 \%$ \\
\hline Number of lenses & 4 & 6 & 5 & 4 \\
\hline Glass-air interfaces (incl. fiber) & 9 & 5 & 5 & 3 \\
\hline Pupil diameter [mm] & $1.223 \mathrm{~mm}$ & $2.503 \mathrm{~mm}$ & $1.827 \mathrm{~mm}$ & $1.280 \mathrm{~mm}$ \\
\hline Pupil diameter variation with wavelength & $0.5 \%$ & $1.3 \%$ & $1 \%$ & $4.6 \%$ \\
\hline
\end{tabular}

\section{OPTICS MANUFACTURING}

\subsection{Lenses}

We procured several sets of the lenses according to design $\mathrm{C} 3$, to assemble the module in-house. The doublets have a diameter of $6 \mathrm{~mm}$, and the singlet $5 \mathrm{~mm}$, to aid mounting with lens spacers. The lenses were manufactured to tight tolerances by BMV optical technologies. Typical error achieved on the singlet lens were better than lambda/10 PV surface irregularity, $<0.2 \%$ radius error, $<1$ arcmin wedge, $<25$ micron center thickness error, $<5$ micron diameter error. The glass-air interfaces were coated with a broadband antireflection coating with less than $1 \%$ average reflectivity across the full wavelength range.

\subsection{Fused silica fiber puck}

Inside the NEID cryostat the five fibers ( $3 \mathrm{HR}$ and $2 \mathrm{HE}$ ) in the fiber bundle are potted inside a custom designed fused silica puck made by Femtoprint. Fused Silica was used for the puck since the fiber core is also fused silica; the matching coefficient of thermal expansion reduces stresses from compression of the puck as the instrument goes from room temperature to its operating temperature of $300 \mathrm{~K}$. The puck has five boreholes sized to the NEID fibers and has conical openings leading to each fiber to help ease the fibers into the bores. The V-groove on the puck is to help clock the puck and lock it in place inside its mount. The procedure followed to epoxy the puck is similar to that followed for the HPF puck as has been detailed in Kanodia et al. $(2018)^{8}$.

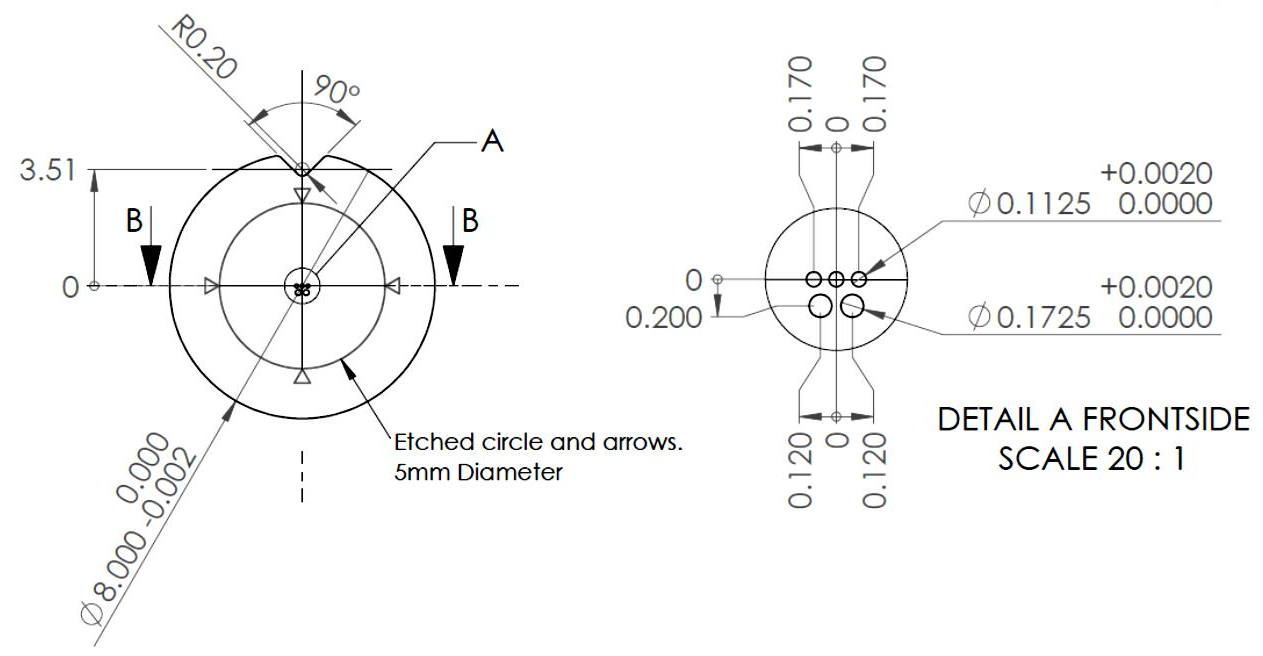

Figure 11. Manufacturing drawing of the fused silica fiber puck, with a detail view of the bores that hold the three HR and the two HE fibers. 

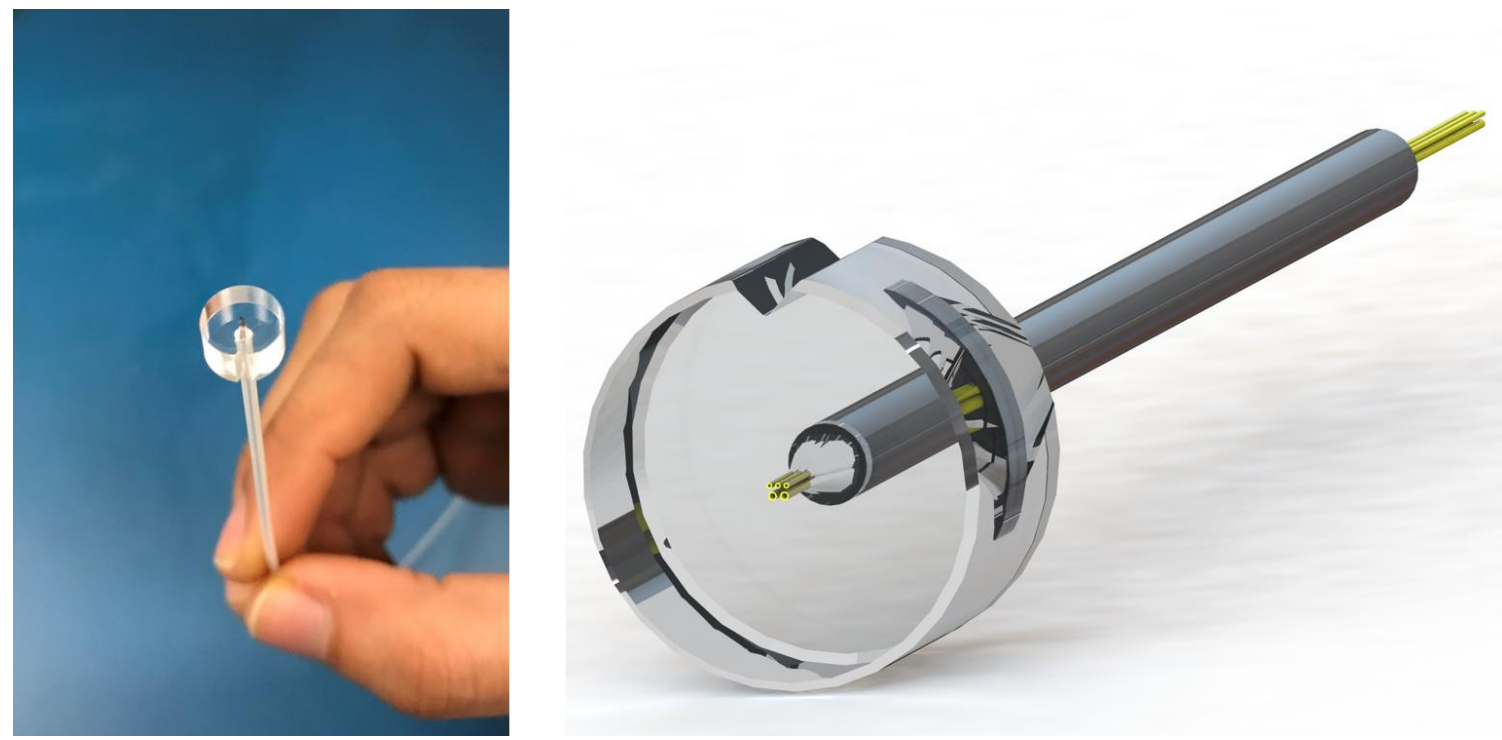

Figure 12. Picture and CAD rendering of the fiber puck.

\section{LENS TUBE MECHANICAL DESIGN}

The lens tube was machined out of Aluminium 6061 to match expansion coefficients of the NEID bench and optical mounts. The parts were manufactured by Hilltop Technology Laboratory, Inc. Because the centering tolerance was tight, we opted for black anodized (Anodize MIL-A-8625 Type I, Class 2 Black), since that does not change the dimensions significantly. After anodizing we precision reamed the tube to final tolerances. To assemble the tube, the first doublet is inserted into the tube until it bottoms out, then an aluminium spacer is installed followed by the next doublet and another spacer. The fiber puck face is inserted into the final spacer and references against the face of the spacer. Then a horseshoe shaped aluminium plunger is installed and with the help of a threaded cap and wave disc spring compresses the entire internal assembly and provides some compliance when the tube expands to operating temperature. A cone tip set screw provides clocking constraint on the fiber puck which has a groove along its edge. The spacers are threaded internally to act as light baffles and also anodized. A small aperture was machined in the L1 spacer to prevent some back reflections from L2 onto L1.

A cylindrical spacer at the end of the tube allows for focus adjustment to mount the lens tube parfocal with a single mode fiber that was used for focus tests. Once the tube is clamped in place, the front plate and cylinder spacer are removed. The top clamp is machined with an included angle of 4 degrees to clock the rotation of the tube so that the final image is orthogonal on the CCD plane after reflection off the grating. Five tubes were constructed, each were individually assembled and spacers for each assembly were individually modified in length to achieve parfocal performance. Each fiber bundle assembly and lens tube assembly were ranked and paired together. The best pair was installed and the remaining are spares.

The lens tubes and fiber assemblies were assembled in the clean room and tested outside of the instrument. In order to install the assembled lens tube in the instrument, the lens tube diameter had to be smaller than $15 \mathrm{~mm}$ to fit through the vacuum feedthrough. The lens tube and spacer each also have a small hole drilled into their sides to provide an air purge path. 


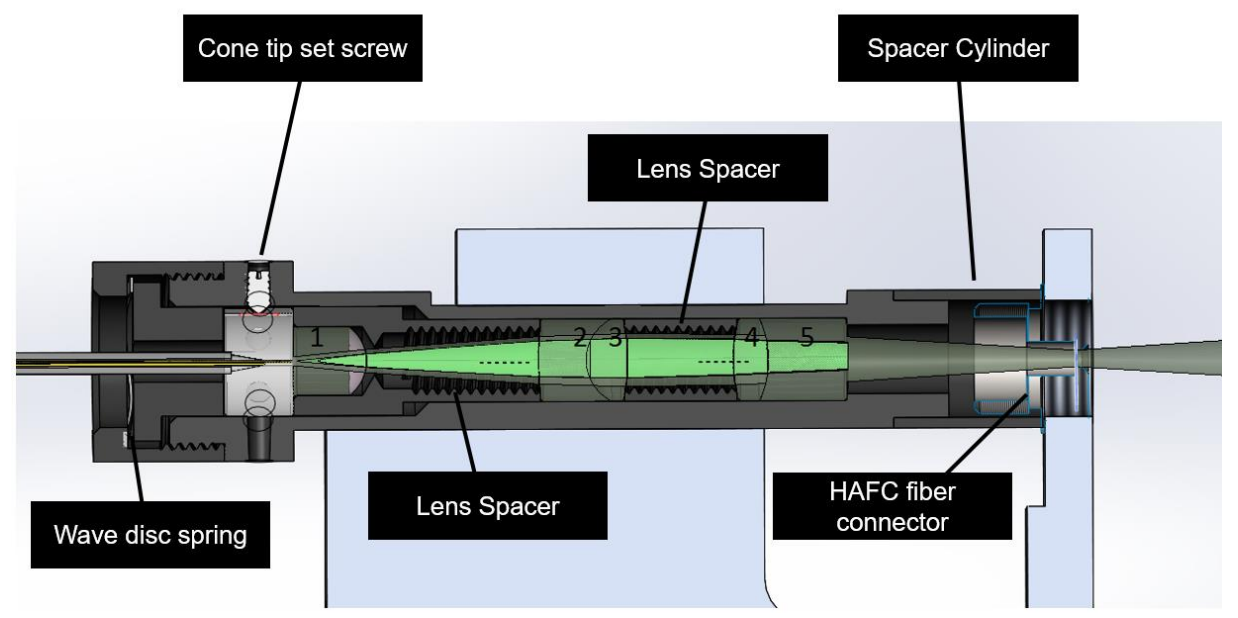

Figure 13. Cross sectional view of the fiber injection optics in their lens tube.

\section{SUMMARY}

This paper describes the trade-off study for the fiber injection optics of the NEID precision radial velocity spectrograph. The optics convert the $\mathrm{f} / 3.6$ beam from the multimode fibers to f/7.95, matched to NEID's collimator mirror. We discussed the technical requirements and the design process of the optical system, and have shown the system's performance. The final design, selected for a good compromise between wavefront error, throughput and complexity, uses two cemented doublets and a singlet lens in contact with the fiber aperture to generate a relay with a real focus. The optics produce diffraction limited performance over the $0.25 \mathrm{~mm}$ object field across the full wavelength range of the spectrograph, from 380 to $930 \mathrm{~nm}$. The optics were assembled in house into a precision aluminium lens tube, together with a custom-made fused silica puck that holds the five science, sky and calibration fibers feeding NEID.

\section{REFERENCES}

[1] Schwab, C., Rakich, A., Gong, Q., Mahadevan, S., Halverson, S. P. et al., "Design of NEID, an extreme precision Doppler spectrograph for WIYN", Proc. SPIE 9908, id. 99087H 6 pp (2016)

[2] Baranne, A., "White Pupil Story or Evolution of a Spectrographic Mounting," Very Large Telescopes and their Instrumentation, ESO Conference and Workshop Proceedings 2, 1195-1206 (1988).

[3] Schwab, C., Liang, M., Gong, Q., bender, C., Blake, C., Halverson, S., et al., "The NEID precision radial velocity spectrometer: optical design of the port adapter and ADC", Proc SPIE 10702, id. 1070271 (2018)

[4] Halverson, S., Terrien, R., Mahadevan, S., Roy, A., Bender, C. et al., "A comprehensive radial velocity error budget for next generation Doppler spectrometers", Proc. SPIE 9908, id. 99086P 20 pp. (2016)

[5] Mahadevan, S., Halverson, S., Ramsey, L. and Venditti, N. "Suppression of fiber model noise induced radial velocity errors for bright emission-line calibration sources," The Astrophysical Journal 786, 18-24 (2014).

[6] Halverson, S., Roy, A., Mahadevan, S., Ramsey, L., Levi, E., Schwab, C., Hearty, F., MacDonald, N., “An Efficient, Compact, and Versatile Fiber Double Scrambler for High Precision Radial Velocity Instruments", The Astrophysical Journal 806(1), 61 (2015)

[7] Stuermer, J., Stahl, O., Schwab, C., Seifert, W., Quirrenbach, A., et al., "CARMENES in SPIE 2014. Building a fibre link for CARMENES”, Proc. SPIE 9151, id. 915152 (2014)

[8] Kanodia, S., Mahadevan, S., Ramsey, L., et al, "Overview of the spectrometer optical fiber feed for the Habitablezone Planet Finder”, Proc SPIE 10702, id. 107026Q (2018) 